\title{
AVALIAÇÃO DA INÉRCIA ROTACIONAL DE CADEIRA DE RODAS MANUAL: implicações para o design ergonômico
}

\author{
Fausto Orsi Medola \\ Departamento de Design e Programa de Pós-graduação em Design, FAAC, UNESP, \\ Bauru-SP. \\ fausto.medola@faac.unesp.br \\ Stephen Sprigle \\ Center for Assistive Technology and Environmental Access, School of Industrial \\ Design, Georgia Institute of Technology, Atlanta, EUA. \\ stephen.sprigle@coa.gatech.edu
}

Resumo: No design de uma cadeira de rodas manual, o desempenho na locomoção é um dos aspectos de maior preocupação, em vista da importante limitação de mobilidade à qual os usuários estão expostos. 0 desempenho na mobilidade é resultante, basicamente, do equacionamento entre os esforços do usuário, os aspectos inerciais da cadeira de rodas e a resistência ao movimento (principalmente no atrito entre as rodas e a superfície). O designer é capaz de intervir nesta equação - e desta forma no desempenho da mobilidade - abordando os aspectos inerciais do equipamento. Para deslocamentos em trajetória retilínea, a massa total da cadeira de rodas é o principal componente inercial. Por outro lado, para movimentos em trajetórias curvas ou mistas, bem como para manobras de giro da cadeira, assume grande relevância a inércia rotacional do sistema. Neste sentido, o objetivo deste estudo é avaliar a influência da adição de massa (e sua localização) e do uso de dois diferentes conjuntos de aro-roda na inércia rotacional de uma cadeira de rodas manual. Uma plataforma construída com sensores de força e encoder óptico foi utilizada para avaliação da inércia rotacional da cadeira de rodas. As medidas foram realizadas em três situações, em todas sendo utilizada a mesma cadeira de rodas: (i) adição de pesos nas rodas e no centro do assento da cadeira; (ii) dois diferentes conjuntos de rodas e pneus traseiros comercialmente disponíveis; (iii) duas posições - anterior e posterior - do eixo das rodas traseiras. Os resultados demonstram que, para uma mesma configuração de cadeira de rodas, a adição de corpos de pouca massa $(0,25 \mathrm{~kg}$ e $0,4 \mathrm{k})$ junto aos eixos das rodas traseiras provoca maior aumento da inércia rotacional do que a adição de $5,5 \mathrm{~kg}$ em posição centralizada. Similarmente, o conjunto de rodas de maior massa resultou em maior inércia rotacional, assim como a posição posterior das rodas (consequentemente maior comprimento da cadeira). Este estudo contribui 
com duas diretrizes projetuais para cadeira de rodas: a massa do equipamento deve estar concentrada, o máximo possível, próximo ao centro de massa da cadeira; as medidas de comprimento e largura devem ser as menores possíveis, de modo a diminuir as distâncias dos extremos (e suas massas) anterior, posterior e lateriais para o centro de massa do equipamento. Designers devem, no processo de desenvolvimento de cadeiras de rodas manuais, considerar os aspectos inerciais do equipamento, de forma a favorecer o desempenho da mobilidade de seus usuários e, assim, sua funcionalidade, independência e qualidade de vida.

Palavras-chave: Cadeira de rodas, Mobilidade, Design, Tecnologia Assistiva.

Abstract: In the design of a manual wheelchair, the performance during mobility is one of the aspects of great concern, due to the important limitation in the mobility among users. The mobility performance results, basically, from the equation between user's manual efforts, wheelchair inertial properties and rolling resistance (mainly the friction between the wheels and the surface). The designer can intervene in this equation - and thus in the mobility performance - by addressing the inertial aspects of the equipment. For straight-forward displacements, wheelchair total mass is the main inertial component. In contrast, for turning trajectories and maneuvers, rotational inertia of the system plays an important role. The aim of this study was to evaluate the influence of adding mass (and its location) and the use of two different sets of rear wheels on the rotational inertia of a manual wheelchair. A platform instrumented with force sensors and optical encoder was used to measure the rotational inertia of the wheelchair. Measurements were taken in three different situations (all them with the same wheelchair): (i) addition of mass in the wheels and in the center of the seat; (ii) two different sets of commercially available rear wheels; (iii) two positions - rearward and forward - of the rear wheels' axle. The results show that, for the same wheelchair configuration, the addition of low weight bodies $(0.25 \mathrm{~kg} \mathrm{e} 0.4 \mathrm{k})$ in the rear wheels led to a greater increase in the rotational inertia of the system than adding $5.5 \mathrm{~kg}$ in the center of the seat. Similarly, the heavier set of rear wheels resulted in greater rotational inertia, as well as the rearward position of the rear wheels axle. This study contributes with two ergonomics guidelines for the design of manual wheelchairs: the equipment mass should be concentrated, as possible, close to the center of mass of the chair; the wheelchair length and width should be as small as possible, in order to reduce the distances to the wheelchair center of mass. In the design process of manual wheelchairs, designers should consider the inertial aspects of the equipment, in order to improve mobility performance and, thus, benefiting users' functionality, independence and quality of life.

Keywords: Wheelchairs, Mobility, Design, Assistive Technology. 


\section{INTRODUÇÃO}

A locomoção é uma característica essencial do ser humano, necessária para o desempenho da maioria das atividades de vida diária. Para muitas pessoas com limitação da mobilidade, a cadeira de rodas é o dispositivo de assistência através do qual a locomoção pode ser desempenhada. No entanto, comparada a outras formas de locomoção, a propulsão manual de cadeira de rodas é um modo altamente ineficiente de locomoção (VAN DER WOUDE et al., 2001).

Em sua forma mais tradicional, a cadeira de rodas manual, o usuário se locomove por meio da aplicação de forças (impulsões) nas rodas traseiras de forma rítmica, e a intensidade, simetria e frequência com as quais tais forças são aplicadas determinam a velocidade e trajetória do movimento. A partir de uma perspectiva mecânica, o movimento resultante representa o equacionamento entre as ações do usuário, os aspectos inerciais do equipamento e a resistência ao movimento das rodas (SPRIGLE, 2009). Neste sentido, o design e configuração da cadeira de rodas assumem grande relevância, pois afetam os aspectos inerciais e, como resultado, a atuação do usuário na locomoção.

Este estudo tem como objetivo avaliar a influência do design e configuração de cadeiras de rodas manuais na inércia rotacional (IR) do sistema. Este conhecimento possibilita, em última análise, compreender de que forma a atuação do usuário pode ser influenciada pelo design e configuração do equipamento. Contribui, portanto, para a atividade de designers, fabricantes e demais profissionais que trabalham diretamente com o projeto e produção de cadeira de rodas, a fim de que os produtos desenvolvidos ofereçam melhores condições de mobilidade aos usuários.

\section{FUNDAMENTAÇÃO TEÓRICA}

\subsection{Problemas ergonômicos na mobilidade em cadeiras de rodas manuais}

Estudos apontam alta prevalência de dor nos membros superiores entre usuários de cadeira de rodas manual, especialmente nos ombros e punhos (ALM et al., 2008; CURTIS et al., 1999; SUBBARAO et al., 1994). A natureza repetitiva da atividade de propulsão manual tem sido apontada como um dos fatores de sobrecarga nos membros superiores de usuários de cadeira de rodas (MIYAHARA et al., 1998; AMBROSIO et al., 2005; BONINGER et al., 2004).

Para um usuário de cadeira de rodas manual, as consequências das lesões de membros superiores podem ser incapacitantes, uma vez que a locomoção, transferências da cadeira e a maioria das demais atividades da rotina diária são desempenhadas com os braços e mãos. a preservação da função dos membros superiores é condição essencial para a independência funcional dos usuários de cadeira de rodas (PARALYZED VETERANS OF AMERICA CONSORTIUM FOR SPINAL CORD MEDICINE, 2005). Desta forma, é importante que o design de cadeiras manuais busque reduzir ao máximo a sobrecarga biomecânica na propulsão manual e aprimorar a eficiência na locomoção.

Possivelmente devido à elevada carga biomecânica relacionada à atividade de propulsão manual, os usuários de cadeira de rodas têm importantes limitações na locomoção independente por longas distâncias, rampas e em terrenos com aclives. Nestas situações, mover-se sem a ajuda de terceiros representa um desafio 
extenuante, difícil e, por vezes, impossível de ser superado. Recentes estudos possibilitam a compreensão do impacto da limitação de mobilidade na rotina diária: a média de distância percorrida em um dia, através da marcha normal, por adultos sem deficiência é de 6 a 7,5 km (BOHANNON, 2007), enquanto que usuários de cadeira de rodas percorrem de 1,5 a 2,5 km (KARMARKAR et al., 2010; LEVY et al., 2010; TOLERICO et al., 2007).

Para os profissionais que trabalham com o design, configuração e fabricação de cadeira de rodas, talvez mais importante do que saber o quanto as pessoas se movem seja entender como se movem. Esta questão é essencial para se compreender de que forma o design da cadeira de rodas pode afetar a mobilidade de seu usuário, assim contribuindo para o desenvolvimento de produtos mais adequados ao modo de utilização, necessidades e expectativas dos usuários. Embora o tempo de ocupação diária da cadeira seja relativamente longo (aproximadamente 11 horas/dia), em apenas aproximadamente $10 \%$ do tempo os usuários estão efetivamente em movimento (Sonenblum et al., 2012). Sonenblum et al. (2012) estudaram a mobilidade em cadeira de rodas, e encontraram que a locomoção na rotina diária dos usuários é caracterizada principalmente por pequenos e numerosos "episódios de movimento" (aproximadamente distância de 8,6 metros, duração de 21 segundos e velocidade de $0,43 \mathrm{~m} / \mathrm{s}$ )

\subsection{A locomoção em cadeira de rodas manual}

A dinâmica de uma cadeira de rodas durante a locomoção diária é influenciada pelas mudanças inerciais durante o início, a frenagem e as curvas. A partir de uma perspectiva mecânica, dois fatores influenciam de forma importante o movimento de uma cadeira de rodas manual: o atrito e a inércia. Tais fatores são manifestados como resistência ao rolamento das rodas, atrito dos rolamentos, massa total e a distribuição de massa, entre outros (SPRIGLE, 2009). Desta forma, as forças manuais aplicadas pelo usuário nos aros propulsores interagem com o atrito e as forças inerciais, e o resultado desta equação de forças é o movimento da cadeira de rodas que, fisicamente, é representado pela sua energia cinética.

Uma cadeira de rodas manual pode ser entendida como a composição de sete corpos rígidos distintos conectados de tal forma a possibilitarem a mobilidade: o quadro, as duas rodas traseiras, as duas pequenas rodas dianteiras (casters) e os dois garfos que suportam, respectivamente, as rodas dianteiras (Figura 2). 0 desempenho da cadeira de rodas durante a locomoção depende, em grande parte, dos aspectos inerciais de cada componente e da configuração destes no sistema (cadeira de rodas). 


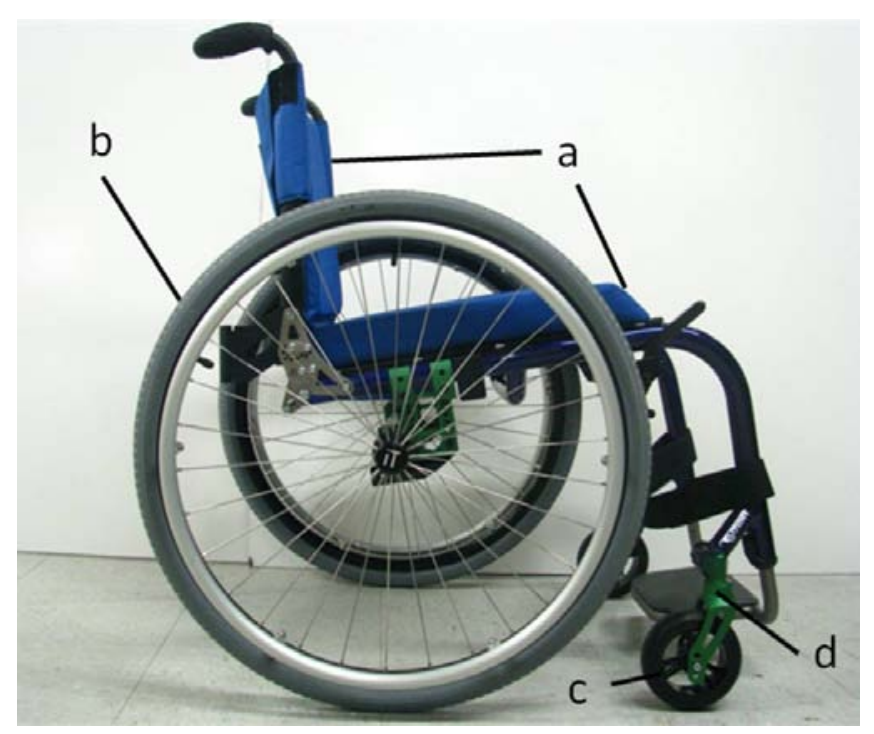

Figura 2. Sete componentes essenciais de uma cadeira de rodas: (a) quadro; (b) rodas traseiras; (c) rodas dianteiras (casters); (d) suporte ou "garfo" dos casters. Fonte: Elaborado pelo autor, com base na pesquisa realizada.

Do ponto de vista mecânico, para movimentos em trajetória retilínea, o aspecto inercial mais importante é a massa total do sistema. Neste sentido, quanto maior a massa total da cadeira, tanto maior será a dificuldade do usuário em impor acelerações e desacelerações - tais como ao iniciar e frear - no movimento. Por outro lado, quando a trajetória é curvilínea ou mista (alternância de direções do movimento), a inércia de cada um dos componentes e suas posições relativas ao centro de massa na geometria do sistema assumem papel importante, pois determinam a inércia rotacional (IR) do sistema (MEDOLA et al. 2014a). A IR (ou momento de inércia) é uma medida da resistência que um corpo oferece ao movimento de rotação, sendo o análogo rotacional da massa no movimento linear. Desta forma, é dependente da massa e do raio do objeto, conforme demonstrado abaixo:

$$
\mathrm{I}=\mathrm{m} \cdot \mathrm{r}^{2}
$$

Na prática, isto sugere que quanto maior a massa das rodas traseiras, maior será o esforço do usuário para impor à cadeira uma trajetória curva. Considerando que a locomoção em cadeira de rodas na vida diária compreende pequenos deslocamentos e constante alternância de direção da trajetória (Sonemblum et al., 2012), a distribuição de massa no equipamento é um fator tão ou mais importante do que a massa total do sistema.

A atuação do usuário sobre a cadeira de rodas manual ocorre por meio das forças aplicadas ao aro propulsor (tubo cilíndrico metálico acoplado às rodas traseiras), em um padrão rítmico que define duas fases: toque (quando o usuário tem suas mãos em contato com os aros propulsores); e fase de recuperação, quando o usuário libera as mãos dos aros e as balanceia para trás, com o objetivo de alcançar novamente os aros e iniciar novo ciclo propulsor.

Portanto, o usuário consegue alterar a direção do movimento somente quando aplica nestes forças assimétricas. Durante a fase de recuperação, quando as mãos são 
liberadas dos aros, a cadeira de rodas assume trajetória retilínea. Pode-se concluir, a partir do exposto acima, que a locomoção em trajetória curva compreende a alternância entre trajetória curva e retilínea. Para manter a cadeira em uma trajetória curva, o usuário "luta" para alterar a direção do movimento de uma cadeira cuja tendência é deslocar-se em linha reta. Nesta situação, tanto a massa das rodas traseiras quanto a distância destas para o centro de massa do equipamento determinam a dificuldade do usuário em girar a cadeira ou mover-se por uma trajetória curva.

O presente estudo tem como objetivo avaliar a influência da massa, distribuição de massa e configuração da cadeira de rodas na inércia rotacional do sistema. Este conhecimento pode contribuir no entendimento dos aspectos inerciais de uma cadeira de rodas, bem como a importância de se considerar esta visão mecânica e ergonômica no design, configuração e fabricação de cadeira de rodas.

\section{MATERIAIS E MÉTODOS}

Este estudo foi realizado no Rehabilitation Engineering Applied Research Laboratory (REAR Lab), Center for Assistive Technology and Environmental Access (CATEA), Georgia Institute of Technology, Atlanta, Estados Unidos.

\subsection{Materiais}

A cadeira de rodas utilizada no estudo permite o ajuste da posição horizontal (na direção anterior-posterior) do eixo das rodas traseiras em 44,5 mm. Este ajuste é comumente presente em cadeiras de rodas manuais, e possibilita ao usuário ajustar a posição das rodas de acordo com a estabilidade desejada. Quanto mais à frente as rodas, melhor o alcance das mãos durante o contato com os aros propulsores, porém a cadeira tem menos estabilidade traseira. Uma vez que este ajuste altera o comprimento da cadeira, também foi avaliado no que diz respeito aos efeitos na IR do sistema.

A avaliação da IR de cadeira de rodas foi realizada com a plataforma iMachine (EICHOLTZ et al., 2012), que é um equipamento composto de uma plataforma giratória instrumentada com três células de carga dispostas triangularmente e equidistantes umas às outras e ao centro da plataforma, onde há um encoder ótico (Figura 2). As informações dos sensores de massa (células de carga) e de rotação da plataforma (encoder ótico) são coletadas por um dispositivo de aquisição de dados conectado a um computador, onde os dados são analisados em programa desenvolvido em ambiente Labview 7.0 (National Instruments, Austin, TX, USA). 


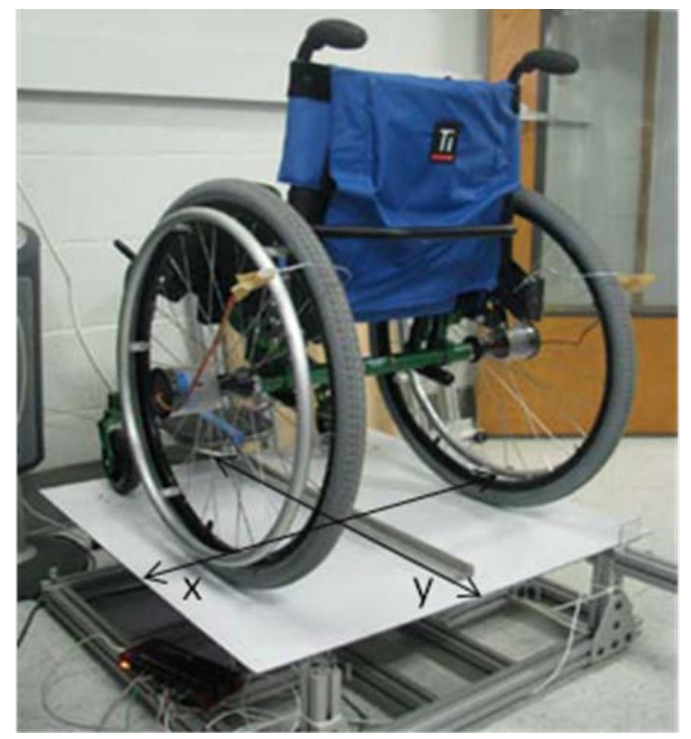

Figura 2. iMachine - equipamento para medição da inércia rotacional de cadeira de rodas: oscilação da plataforma em eixo normal à intersecção das coordenadas $x$ e $y$. Fonte: Elaborado pelo autor, com base na pesquisa realizada.

\subsection{Métodos}

O procedimento de avaliação consiste em posicionar a cadeira de rodas sobre no centro plataforma, o que é facilitado pela visualização em tempo real das massas registradas pelas três células de carga, permitindo assim ao operador ajustar a posição da cadeira até que a condição ideal seja atingida.

Após garantir o posicionamento da cadeira de rodas no centro da plataforma, uma alavanca é acionada para produzir um deslocamento na plataforma que, após liberada, oscilada em torno de um eixo que cruza verticalmente o centro da plataforma, em rotação com frequência aproximada de 1,25 Hz. A partir das medidas de massa e período de oscilação da plataforma, obtém-se a inércia rotacional do sistema. Para cada medida, este procedimento foi repetido por cinco vezes, e a média dos valores medidos foi então calculada.

Em todos os testes, foi utilizada a mesma cadeira de rodas (modelo Aero Z, TiLite). No entanto, com o objetivo de comparar os efeitos de diferentes configurações de cadeira de rodas na IR do sistema, dois diferentes pares de rodas traseiras foram utilizados (Figura 3). Os efeitos da posição horizontal do eixo das rodas também foi investigada, pois a cadeira utilizada permiteo ajuste horizontal (anterior-posterior) das rodas traseiras em 44,4 $\mathrm{mm}$. Ainda, foi avaliado o efeito da adição de corpos de diferentes pesos em nas rodas e no centro do assento na IR do sistema. 


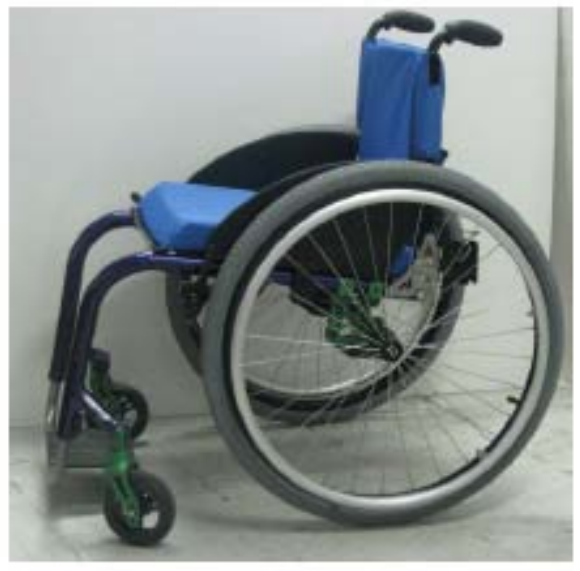

(a)

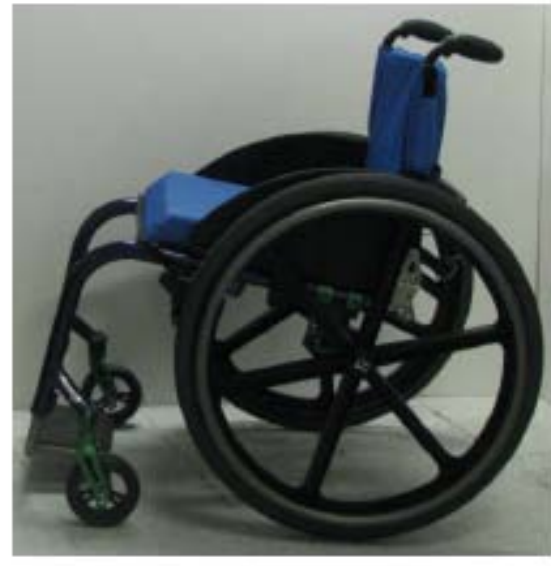

(b)

Figura 41. Dois modelos de conjunto pneu-roda: (a) pneu com enchimento a ar e roda multi-raios; (b) pneu maciço e roda com raios largos. Fonte: Elaborado pelo autor, com base na pesquisa realizada.

Para apreciação dos resultados, são apresentadas as médias das cinco medidas realizadas da IR para cada configuração da cadeira de rodas.

\section{RESULTADOS}

A análise dos efeitos do acréscimo de corpos de diferentes pesos nas rodas ou em posição central do assento da cadeira de rodas demonstra que, de uma forma geral, a distribuição de massa é mais determinante para a IR da cadeira de rodas do que propriamente sua massa total. Conforme demonstra a Tabela 1, o acréscimo de $0,25 \mathrm{~kg}$ no eixo de cada roda traseira resultou em maior aumento da IR $(5,9 \%)$ do que o acréscimo de $5,5 \mathrm{~kg}(2,36 \%)$ no centro do assento.

Tabela 1 - Efeitos da adição de corpos de diferentes massas em diferentes posições na IR do sistema

\begin{tabular}{|c|c|c|c|}
\hline $\begin{array}{c}\text { Configuração da Cadeira de } \\
\text { Rodas }\end{array}$ & $\begin{array}{l}\text { Massa Total } \\
\quad(\mathrm{kg})\end{array}$ & $\begin{array}{l}\text { IR média } \\
\left(\mathrm{kg} \cdot \mathrm{m}^{2}\right)\end{array}$ & $\begin{array}{c}\text { Porcentagem de aumento } \\
\text { em relação à } \\
\text { configuração original }\end{array}$ \\
\hline $\begin{array}{l}\text { Cadeira de rodas com } \\
\text { configuração original }\end{array}$ & 12,15 & 1,101 & - \\
\hline $\begin{array}{c}\text { Cadeira de rodas }+0,25 \mathrm{~kg} \text { no } \\
\text { eixo de cada roda traseira }\end{array}$ & 12,65 & 1,166 & $5,9 \%$ \\
\hline $\begin{array}{l}\text { Cadeira de rodas }+0,4 \text { kg no } \\
\text { eixo de cada roda traseira }\end{array}$ & 12,95 & 1,201 & $9 \%$ \\
\hline $\begin{array}{l}\text { Cadeira de rodas }+1,2 \mathrm{~kg} \text { no } \\
\text { centro do assento }\end{array}$ & 13,35 & 1,102 & $0,09 \%$ \\
\hline $\begin{array}{l}\text { Cadeira de rodas }+5,5 \mathrm{~kg} \text { no } \\
\text { centro do assento }\end{array}$ & 17,65 & 1,127 & $2,36 \%$ \\
\hline
\end{tabular}

A mudança de configuração de uma mesma cadeira de rodas, ou seja, a posição do eixo das rodas traseiras e o conjunto roda/pneu utilizado - também afetou a IR do sistema (Tabela 2). Enquanto a posição do eixo das rodas traseiras modifica a 
geometria mantendo-se a massa total da cadeira, o conjunto pneu/roda altera a distribuição de massa, em uma mesma geometria. Considerando ambas as situações, o menor valor de IR foi encontrado com a configuração que combinou a posição anteriorizada do eixo das rodas traseiras e os pneus a ar / rodas multi-raios (1,073 kg$\mathrm{m}^{2}$ ). Por outro lado, a combinação entre posição posteriorizada do eixo das rodas traseiras e pneus maciços / rodas raio largo apresentou a maior IR, 11\% maior em relação à configuração de menor IR.

Tabela 2 - Influência do conjunto pneu / roda e da posição das rodas traseiras na IR do sistema

\begin{tabular}{ccccc}
\hline $\begin{array}{c}\text { Posição do eixo } \\
\text { das rodas traseiras }\end{array}$ & Conjunto Pneu / roda & $\begin{array}{c}\text { Massa } \\
\mathbf{( k g )}\end{array}$ & $\begin{array}{c}\text { IR Média } \\
\mathbf{( k g . \mathbf { 2 } ^ { 2 } )}\end{array}$ & $\begin{array}{c}\text { Porcentagem de } \\
\text { aumento em } \\
\text { relação à menor } \\
\text { IR }\end{array}$ \\
\hline Anterior & Pneus a ar / Rodas multi-raios & 12,15 & 1,073 & - \\
\hline Posterior & Pneus a ar / Rodas multi-raios & 12,15 & 1,101 & $2,6 \%$ \\
\hline Anterior & Pneus maciços / Rodas raio largo & 13,30 & 1,169 & $8,94 \%$ \\
\hline Posterior & Pneus maciços / Rodas raio largo & 13,30 & 1,191 & $11 \%$ \\
\hline
\end{tabular}

\section{DISCUSSÃO}

O presente estudo demonstrou que o design e configuração de cadeiras de rodas manuais determinam a inércia rotacional de um equipamento e, portanto, afetam as cargas impostas ao usuário durante a locomoção e a dirigibilidade da cadeira. Ainda, os resultados demonstram que a distribuição de peso na geometria da cadeira de rodas exerce maior influência na IR da cadeira de rodas do que sua massa total sendo, portanto, um aspecto de atenção para designers e fabricantes de dispositivos de mobilidade sentada.

Os resultados deste estudo devem ser compreendidos a partir da perspectiva das implicações do design e configuração da cadeira de rodas na mobilidade do usuário. Especificamente, a IR do sistema afeta a dirigibilidade do dispositivo, em especial a realização de manobras de giro sobre o próprio eixo e a locomoção e trajetórias curvas ou mistas. Os movimentos em trajetória curvilínea merecem destaque, uma vez que são caracterizados pela alternância entre giro da cadeira e movimento retilíneo. Durante o deslocamento em trajetória curva, o usuário consegue fazer com que a cadeira gire apenas quando aplica forças assimétricas nas duas rodas. A partir do momento que libera as mãos do aro (movimento necessário para iniciar um novo ciclo propulsor), a cadeira assume movimento retilíneo, conforme demonstrado em um recente estudo (MEDOLA et al., 2014a). Portanto, nesta situação, quanto maior a IR do sistema, maior a dificuldade do usuário em retirar a cadeira de uma movimento retilíneo e assumir trajetória curva.

Os resultados do presente estudo podem ser correlacionados com o estudo de MEDOLA et al. (2014a), que demonstraram que, para movimentos em trajetória retilínea, o principal componente inercial da energia cinética é a massa total da cadeira e sua velocidade em deslocamento translacional. Em outras palavras, para 
deslocamentos em trajetória retilínea, a energia cinética em rotação (giro da cadeira ou movimento curvilíneo) é desprezível e, portanto, a massa total da cadeira é o principal componente inercial. Por outro lado, para manobras de giro da cadeira sobre o próprio eixo, o principal componente inercial da energia cinética refere-se à inércia rotacional do sistema que, conforme demonstrado no presente estudo, decorre diretamente e é mais dependente da distribuição de massa na geometria do sistema do que de sua massa total.

O processo de desenvolvimento de uma cadeira de rodas manual, no âmbito do design ergonômico, envolve a concepção de um dispositivo de mobilidade para pessoas com deficiência, de forma a reduzir as cargas biomecânicas e aumentar a eficiência da locomoção e, desta forma, favorecer a funcionalidade e independência do usuário. Os diversos aspectos do design e configuração de cadeira de rodas manual que afetam a mobilidade foram discutidos em um recente estudo (MEDOLA et al., 2014b). É também objetivo do designer conceber um produto cujo uso proporcione conforto e satisfação. No entanto, implementar todas estas qualificações em um único projeto representa um desafio de difícil resolução plena durante a prática projetual. Um exemplo: para melhorar o conforto na postura sentada do usuário de cadeira de rodas, a solução mais imediata seria aumentar as dimensões das superfícies de suporte (assento, encosto, apoio para os pés, apoio para os braços), de modo a distribuir o peso do corpo por uma maior área e, desta forma, reduzir a pressão em pontos específicos da interface usuário-cadeira. No entanto, tal solução implica em aumento da massa e alteração da distribuição de massa do sistema. O uso de apoio de braços, em vista de sua localização nos extremos laterais do assento, aumenta a concentração de massa nesta região e, portanto, implica em maior IR do sistema, conforme demonstrado no presente estudo. Além disso, um estudo demonstrou que a posição do apoio dos pés influencia a IR do sistema e está diretamente relacionada ao desempenho na realização de manobras de giro da cadeira de rodas (MACPHEE et al., 2001).

As implicações práticas para o design e configuração de cadeiras de rodas manuais podem ser resumidas em três recomendações: (i) a massa total da cadeira de rodas deve ser a menor possível; (ii) a distribuição de massa na geometria da cadeira deve buscar concentrar a maior parte da massa próxima ao centro de massa do sistema; (iii) a cadeira de rodas deve possuir menores medidas de comprimento e largura possíveis (medidas nas direções anteroposterior e látero-lateral), reduzindo assim a distância dos extremos da cadeira para o centro do equipamento. É importante ressaltar que designers e fabricantes devem considerar que estas recomendações não podem ser compreendidas como regra geral para um projeto de cadeira de rodas, uma vez que influenciam outros aspectos da interação entre usuário e equipamento, tais como a estabilidade, segurança e conforto do usuário. No entanto, se consideradas como diretrizes, podem contribuir de forma importante no desenvolvimento de um produto que ofereça condições ótimas de desempenho na mobilidade.

Apesar dos resultados demonstrarem o impacto das alterações na configuração da cadeira de rodas na IR do sistema, os mesmos não indicam a extensão com a qual a mobilidade do usuário é afetada. Apenas a partir de testes que avaliem os aspectos fisiológicos e biomecânicos da locomoção em cadeira de rodas manual e a percepção 
de esforço do usuário nas diversas trajetórias e manobras, será possível verificar as correlações objetivas entre a configuração do equipamento e as reais implicações à demanda de trabalho do usuário.

\section{CONCLUSÃO}

Este estudo demonstrou que o design e configuração dos componentes de uma cadeira de rodas manual afetam a inércia rotacional do sistema e, desta forma, sua dirigibilidade. Os resultados apresentados permitem destacar duas conclusões principais: a distribuição de massa na geometria da cadeira de rodas é mais determinante para a inércia rotacional do que a massa total; a posição anteriorposterior das rodas traseiras determina o comprimento da cadeira e, portanto, a inércia rotacional. A partir destas conclusões, podem ser destacadas duas diretrizes para o design de cadeira de rodas, no que diz respeito ao desempenho na mobilidade: concentrar maior parte da massa em posição centralizada, próximo ao centro de massa da cadeira de rodas; reduzir o máximo possível - sem comprometer a estabilidade - as medidas de comprimento e largura da cadeira de rodas. As implicações ao usuário relacionam-se, principalmente, aos desafios inerciais nas diversas situações de mobilidade diária, que compreendem os movimentos de partida, frenagem, acelerações, manobras de giro e deslocamentos em trajetórias mistas (com frequentes mudanças de direção). Futuros estudos devem buscar investigar, sob a perspectiva do usuário, as correlações entre o design e configuração de cadeira de rodas e as implicações biomecânicas e fisiológicas ao usuário. As informações aqui apresentadas contribuem para a atuação de designers no desenvolvimento de equipamentos que priorizem o melhor desempenho na mobilidade, de forma a favorecer a independência, participação comunitária e qualidade de vida dos usuários de cadeira de rodas.

\section{AGRADECIMENTOS}

À Coordenação de Aperfeiçoamento de Pessoal de Nível Superior - CAPES (Processo n. 0810/12-6), pelo suporte financeiro.

\section{REFERÊNCIAS}

ALM, M.; SARASTE, H.; NORRBRINK, C. Shoulder pain in persons with thoracic spinal co rd injury: Prevalence and characteristics. Journal of Rehabilitation Medicine, v.40, p.277-283, 2008.

AMBROSIO, F.; BONINGER, M.L.; SOUZA, A.L.; FITZGERALD, S.G.; KOONTZ, A.M.; COOPER, R.A. Biomechanics and Strength of Manual Wheelchair Users. Journal of Spinal Cord Medicine, v.28, p.407-414, 2005.

BOHANNON, R.W. Number of pedometer-assessed steps taken per day by adults: a descriptive meta-analysis. Physical Therapy, v.87, n.12, p. 1642-1650, 2007.

BONINGER, M.L.; IMPINK, B.G.; COOPER, R.A.; KOONTZ, A.M. Relation between median and ulnar nerve function and wrist kinematics during wheelchair propulsion. Archives of Physical Medicine Rehabilitation, v.85, p.1141-1145, 2004. 
CURTIS, K. A.; DRYSDALE, G. A.; LANZA, R. D.; KOLBER, M.; VITOLO, R. S.; WEST, R. Shoulder Pain in Wheelchair Users with Tetraplegia and Paraplegia. Archives of Physical Medicine and Rehabilitation, v.80, n.4, p.453-457, 1999.

EICHOLTZ, M.R. Design and analysis of an inertial properties measurement device for manual wheelchairs. Master Thesis - Master of Science in the School of Mechanical Engineering. Georgia Institute of Technology, August, 2010, 106 p.

KARMARKAR, A.M.; COLLINS, D.M.; KELLEHER, A.; DING, D.; OYSTER, M.; COOPER, R.A. Manual wheelchair-related mobility characteristics of older adults in nursing homes. Disability and Rehabilitation, v.5, n.6, p. 428-437, 2010.

LEVY, C.E.; BUMAN, M. P.; CHOW, J.W.; TILLMAN, M.D.; FOURNIER, K.A.; GIACOBBI, P. Use of power assist wheels results in increased distance traveled compared with conventional manual wheeling. American Journal of Physical Medicine and Rehabilitation, v. 89, n. 8, p. 625-634, 2010.

MACPHEE, A.H.; KIRBY, R.L.; BELL, A.C.; MACLEOD, D.A. The effect of knee-flexion angle on wheelchair turning. Medical Engineering \& Physics, v.23, p.275-283, 2001.

MEDOLA, F.O.; DAO, P.V.; CASPALL, J.J.; SPRIGLE, S. Partitioning kinetic energy during freewheeling wheelchair maneuvers. IEEE Transactions on Neural Systems and Rehabilitation Engineering, v.22, n.2, p. 326-333, 2014a.

MEDOLA, F.O.; ELUI, V.M.; SANTANA, C.D.; FORTULAN, C.A. Aspects of Manual Wheelchair Configuration Affecting Mobility: A Review. Journal of Physical Therapy Science, v26, n.2, pp. 313-318, 2014b.

MIYAHARA, M.; SLEIVERT, G.G.; GERRARD, D.F. The relationship of strength and muscle balance to shoulder pain and impingement syndrome in elite quadriplegic wheelchair rugby players. International Journal of Sports Medicine, v.19, n.3, pp.210-214, 1998.

PARALYZED VETERANS OF AMERICA CONSORTIUM FOR SPINAL CORD MEDICINE. Preservation of upper limb function following spinal cord injury: A clinical guideline for health-care professionals. Journal of Spinal Cord Medicine, v.28, n.5, p. 434-470, 2005. SONENBLUM, S.E.; SPRIGLE, S.; LOPEZ, R.A. Manual Wheelchair Use: Bouts of Mobility in Everyday Life. Rehabilitation Research Practice, 2012;753165. doi: 10.1155/2012/753165. Epub 2012 Jul 15.

SPRIGLE, S. On "Impact of surface type, wheelchair weight, and axle position on wheelchair propulsion by novice older adults." Archives of Physical Medicine and Rehabilitation, v.90, p.1073-1075, 2009.

SUBBARAO, J. V.; KLOPFSTEIN, M. D.; TURPIN, R. Prevalance and Impact of Wrist and Shoulder Pain in Patients With Spinal COrd Injury. Journal of Spinal Cord Medicine, v.18, n.1, p. 9-13, 1994.

TOLERICO, M.L.; DING, D. COOPER, R.A.; SPAETH, D.M.; FITZGERALD, S.G.; COOPER, R.; KELLEHER, A.; BONINGER, M.L. Assessing mobility characteristics and activity levels of manual wheelchair users. Journal of Rehabilitation Research and Development, v.44, n.4, p.561-571, 2007.

VAN DER WOUDE, L.H.V.; DALLMEIJER, A.J.; JANSSEN, T.W.J.; VEEGER, D. Alternative modes of manual wheelchair ambulation: An overview. American Journal of Physical Medicine and Rehabilitation, v.80, p.765-777, 2001. 